\title{
Histogram Equalization Techniques for Contrast Enhancement: A Review
}

\author{
Sourav Das \\ M.Tech, Final Year Student \\ Department of Electronics \& \\ Communication Engineering, \\ MMU, Mullana (Ambala), \\ Haryana, India
}

\author{
Tarun Gulati \\ Associate Professor \\ Department of Electronics \& \\ Communication Engineering, \\ MMU, Mullana (Ambala), \\ Haryana, India
}

\author{
Vikas Mittal \\ Assistant Professor Department \\ of Electronics \& \\ Communication Engineering, \\ MMU, Mullana (Ambala), \\ Haryana, India
}

\begin{abstract}
Contrast enhancement is one of the widely used techniques for image enhancement. In this technique, contrast of an image becomes better to make the image more acceptable for well human vision. There are several techniques that can be process for contrast enhancement but the most common one is the histogram equalization (HE) for its simplicity. The HE technique remaps gray levels of image according to probability distribution function (PDF). HE spreads the histogram and extends dynamic range of gray levels to accomplish overall contrast enhancement but the drawbacks are excessive change in brightness, excessive contrast enhancement, washed out appearance, loss of naturalness of an image, loss of image details, not displaying the actual appearance of the image so it is not suitable for consumer electronic applications. This paper shows the study of various histogram modifying techniques to overcome these drawbacks in a greater extend.
\end{abstract}

\section{General Terms}

Histogram equalization (HE)

\section{Keywords}

Dynamic histogram equalization, Contrast enhancement Brightness preservation, Histogram partition.

\section{INTRODUCTION}

Image enhancement is one of the most important considerations of digital image processing. Image enhancement focuses on improve the excellence of the input image that may be captured by a camera or other electronics product to make the output image looks better. The enhancement method do not add the extra details in an image, instead they focus on certain specific features of interest in the image. Contrast enhancement is one of the known and famous techniques for image enhancement. It correspondingly enhances visual quality standard by enlarging the dynamic range of gray levels of the input image. It is an important method for the improvement of enhancing video/images in digital processing. HE is a commonly known method for enhancing contrast of an image. HE transforms a uniform distribution of the gray level for an image but then also it is not suitable for consumer electronics as it contains loss of naturalness of an image, loss of image details, washed out appearance and excessive change in brightness as well as excessive contrast enhancement, etc. So to make it consumer reliable and reducing the above drawbacks; some essential operations has to be done on it and these supplementary processes help the HE process to be more effective for giving excellence image quality [1].

\section{ENHANCEMENT TECHNIQUES FOR BETTER CONTRAST}

\subsection{Adaptive Histogram Equalization} (AHE)

One of the famous HE techniques which improve contrast in low contrast images is AHE. It is quite different from other HE techniques with respect to the adaptive method as it generates multiple histograms and each of them responds to a different section of the original image which further requires them to redistribute the brightness level of the image. Therefore, for the whole image representation it requires a single histogram which can be evolved by ordinary HE technique. Thus, AHE is followed up for keeping significant level of improvement in local contrast enhancement and for maintaining the subsequent number of details over the entire image. But it can also generate significant level of noise in an image [2].

\subsection{Contrast Limited Adaptive Histogram Equalization (CLAHE)}

This technique is an advanced form of adaptive histogram equalization. Basically it is invented for providing solutions regarding noise related problems. It works on the small tiles which are very small regions in an image and does not consider the whole image like other traditional methods. Therefore each tile's having contrast enhancement in that manner so that the histograms generated at the output will matches exactly as it is mentioned in the 'Distribution' parameter. Furthermore, adjacent tiles are combined using bilinear interpolation to remove unnecessary local boundaries [3].

\subsection{Brightness Preserving Bi-histogram Equalization (BBHE)}

At first, BBHE split an input image into two sub images based on the mean brightness of the input image. One of the sub images contain set of samples which is less than or equal to the mean and the remaining one is the set of samples greater than the mean. Then the BBHE independently equalizes the sub images histograms with the phenomena that the samples in the formal set are mapped into the range from the minimum gray level to the input mean and the samples in the other set are mapped into the range from the mean to the maximum gray level. In this technique, input mean brightness value is presented by separation intensity $X_{T}$, i.e. the average intensity of all pixels that form the input image. As a result, the mean brightness can be achieved because the original mean brightness is retained [4]. 


\subsection{Dualistic Sub-Image Histogram Equalization Method (DSIHE)}

The DSIHE technique split an original image into two subimages of equal area i.e. one dark and another one is bright. This division of histogram separation is occurred on the bases of the gray level with cumulative probability density equal to 0.5 . Then each of sub images is equalized independently. After the enhancement process, these two sub images are turned into one image. Therefore after sub image HE, the gray level can be sustained in its original scale correspondingly. So, contrast enhancement of the original image is also increased effectively. The theory behind this division is aiming of yielding the maximization of the Shannon's entropy for the output image [5].

\subsection{Recursive Mean Separate Histogram Equalization (RMSHE)}

In this technique, it decomposes the histogram into multi sub histograms rather than two sub histograms in the BBHE. At first, there will be two sub-histograms are obtained on the mean brightness of the original histogram. Thereafter, more sub-histograms are formed on the basis of second and third separating points obtained from mean brightness of two sub histograms. In the same manner, this whole phenomena occurring continuously unless and until required numbers of sub-histograms are met. Later on the HE is performed on each of the sub histogram. Moreover, no desired enhancement is obtained by RMSHE if there will be large number of sub histograms divided [6].

\subsection{Minimum Mean Brightness Error Bi- Histogram Equalization (MMBEBHE)}

It is following the same basic principle of BBHE and DSIHE, i.e. division of original image into two sub images and then applying HE method to equalize resulting sub images separately. While MMBEBHE is a little bit different from them as it searches for a threshold level $1_{t}$, which decomposes the image into two sub images so that it can acquire the minimum brightness difference between the input and the output image. It is called as absolute mean brightness error (AMBE). HE method is performed after the decomposition of input image by the threshold level to generate the output image [7].

\subsection{Brightness Preserving Histogram Equalization with Maximum Entropy (BPHEME)}

It uses a different method to obtain the optimal histogram, which will be the maximum differential entropy stays below the mean brightness preservation limit and then processing the histogram specification according to the instruction of required histogram. Therefore the entropy of the target histogram is maximized below the limit of brightness by applying histogram transformation using optimal brightness preserving enhancement method. The level of gray level is present in small interval in input image and the gray level is stretch in a larger interval in output image. So, they contain the same discrete entropy. Therefore due to its larger dynamic range, the output is enhanced as compared with the input. Mean Absolute Mean Brightness Error (MAMBE) and Entropy are the techniques processed for calculating this result [8].

\subsection{Dynamic Histogram Equalization (DHE)}

The Dynamic Histogram Equalization (DHE) is better than the traditional HE as it enhances an image without any loss of details in the original image. DHE decomposes the global image histogram into a number of sub-histograms based on their local minima and then they will be equalized separately. So gray levels can be mapped by HE because of each sub histogram there will be a dynamic gray level range. A $1 \times 3$ smoothing filter is placed across the image to eliminate the spikes. Therefore to evaluate this, new sub-histograms will have the total available dynamic range of gray levels and it will be based on dynamic range in input image and the cumulative distribution function (CDF) of histogram values. So, small features of the input image are preserved from being washed out and dominated because of this allocation of contrast range stretching. In the end, an independent transformation function is evaluated for each sub-histogram and it will be based on traditional HE. Thereafter output image is mapped according to the input image [9]. Finally, it will be resulting into a moderate contrast enhancement for each and every portion in the image. However this whole technique of DHE can be evaluate into three parts-

i) Partitioning the histogram

ii) Allocating gray level range for each sub histogram

iii) Applying HE on each of them.

\subsection{Weighted Threshold Histogram Equalization (WTHE)}

For image contrast enhancement, this technique is quite fast and effective as it operates on weighted and threshold HE. Thus, weighting and thresholding has been done for modifying the PDF of an image thereafter they processed for HE. So, the original PDF value is modified by weighted and threshold value. Therefore it provides an effective solution to control enhancement process in a convenient way and related to different types of images in an adaptive manner. This whole phenomenon of WTHE depends on modified histogram process. But this technique is failed to preserve the overall brightness of the image and artifacts are also encountered during the enhancement process [10].

\subsection{Recursive sub Image Histogram Equalization (RSIHE)}

In this technique, histogram is divided on the basis of median values (gray level with cumulative probability density equal to $0.5)$ rather than the mean values. However utilization of median values shares the same number of pixels in each partition. Thereafter it divides the histogram into $2{ }^{r}$ pieces of sub histograms and it will preserve the brightness to maximum extent rather than other partitioning methods where $r$ is recursion level and its value is defined by the user. Enhancement performed will be very less if the value of $r$ is greater and this is the serious issue in RSIHE [11].

\subsection{Brightness Preserving Dynamic Histogram Equalization (BPDHE)}

In this method, it can generate output image with mean intensity which is equivalent to the mean intensity of the input image that is basically an extension to the traditional HE method. Therefore it reduces the problems occurred during $\mathrm{HE}$ and preserve the mean brightness of the original image. Then it produces the smoothed histogram of the input image with 1D Gaussian filter and then decomposes the smoothed histogram with respect to its local maximums. Moreover, BPDHE remaps every partition to a new dynamic range 
thereafter HE is done which is quite familiar to DHE. There will be a variation in mean brightness after changing the dynamic range. In the end, it requires the normalization of the output image with respect to the input mean brightness. Thus, resultant output image will carried out the same average intensity as of the input image is having. It requires Average Absolute Mean Brightness Error (AAMBE) for measuring brightness preservation. So, it denotes itself as a well enhancement process and also preserves mean brightness of the image than other traditional HE methods [12].

\subsection{Brightness Preserving Weight Clustering Histogram Equalization (BPWCHE)}

In BPWCHE, it emphasizes on maintaining the overall brightness of the input image and also increases the visualization effect. For separating cluster and evaluating cluster's weight, there will be a non-zero allocation to each bin of the input image. Thus pieces of cluster formation are fully depended on cluster weight, widths of two neighboring clusters and weight ratio. For the evaluation of transformation function for each cluster's sub-histogram, it will require traditional HE methods which are based on new acquired partitions of the result image histogram. Although with the transfer functions, gray level are initialized into the resultant image. Furthermore transformation functions mapped the gray level due to $\mathrm{HE}$ in the enhanced image and the whole phenomena works on distribution of the gray level in the original image. It works well with the contrast enhancement but failed to sustain the brightness [13].

\subsection{Recursive Separated and Weighted Histogram Equalization (RSWHE)}

In this technique, it initially modifies the original histogram and then executes the equalization operation.

This method basically involves three parts:

i) Histogram segmentation module: At first, it generates the histogram of input image. Therefore multiple numbers of sub histograms are generated through the input histogram from their mean and median value.

ii) Histogram weighting module: In this step, sub histograms generated in $1^{\text {st }}$ step will be modified across histogram weighting process by normalized power law function.

iii) HE module: Now HE is performed on each of the modified weighted sub histograms independently. At last, better contrast enhancement is acquired from equalizing sub histogram individually [14].

\subsection{Dynamic Range Separate Histogram Equalization (DRSHE)}

In this technique, it divides the dynamic range of histogram into $\mathrm{k}$ parts and mapped the gray scale levels depends on its area ratio. Thereafter complete uniform HE is done over the resized gray scale level for each sub-histogram. For maintaining the edge of the input image, for effectively histograms variations and for improving overall contrast, it requires Weighted Average of Absolute colour Difference (WAAD). Furthermore, linear adaptive scale factor is required to maintain overall brightness and preserving naturalness of the image so that it enhances visual effect without having any loss of details and without highlighting them. Therefore, it is quite suitable for consumer electronics as well as for displaying applications [15].

\subsection{Adaptively Increasing Value of Histogram Equalization (AIVHE)}

In this technique, original PDF is reshaped for getting a new PDF to overcome the unnecessary changes in the gray level of the image. Therefore it eventually increases the mean brightness of the image. It offers automatic contrast enhancement by adaptive constraint parameter $\alpha(\mathrm{k})$ and it is calculated by the initial value $\gamma$ and user defined parameter $\beta$. It can also provide more gray level distribution for dark and bright regions. Thus, it increases gray level in an effective way. But as it requires human intervention for defining the parameters, it could not be used for personal identification systems [16].

\subsection{Global Contrast Enhancement using Histogram Modification (GCE-HM)}

This technique is quite cheap and having cost reduction function. In this technique, specially designed penalty terms are used and they decide the level of contrast enhancement. It uses a less complex modified histogram algorithm to maintain the enhancement level adaptively for better use of dynamic range as it needs to deal with the noise visibility and naturalness of the image. It emphasizes on noise robustness and overall brightness preservation during the whole process in an effective manner. It also focuses on black/white stretching. This technique is for improving the visual appearances without bothering about specifications in the image. So, it is good for display uses only as contrast enhancement is performed by not including visual artifacts which are generally responsible for degrading the visual quality of the image [17].

\subsection{Image dependent brightness preserving histogram equalization (IDBPHE)}

This technique uses wrapping discrete curvelet transforms (WDCvT) and histogram matching for contrast enhancement and brightness preservation. Curvelet transformations are required to obtain bright regions in the input image. Thereafter with the newly obtained histogram of the brightness regions the original histogram is reshaped. It does not include well preservation of brightness content in an image. It also needs much computation time as it is highly complex because it has to deal with both curvelet and histogram matching techniques [18].

Evaluation of IDBPHE is shown in a precise form:

i) Region Identification and Separation

a. Curvelet transformations are carried out to obtain bright regions in an input image.

ii) Histogram Computation and Matching

a. histogram of the original image is computed with the help of identified brightness regions.

b. original image histogram is modified with respect to the histogram of the identified regions.

\subsection{Quadrants Dynamic Histogram Equalization (QDHE)}

In this technique, it decomposes the histogram into quadrants i.e. four sub-histograms on the basis of the median of the original image. Thereafter, sub-histograms are clipped down depend on the mean of the evolving of the original image before new dynamic range is allocated to each sub-histogram. 
At last, equalization is performed on each sub-histogram. This technique provides enormous results on continuous simulation when compared with traditional HE techniques. So it represents more enhanced images without any noise amplification and over enhancement. Moreover, there will be a complete preservation of image details and highlighting them. This is the reason behind QDHE algorithm for clear images even if they captured in low-light environments and this technique is implementing on the upcoming smartphones. So, it is quite suitable for consumer electronic devices [19].

\subsection{Mean Brightness Preserving Histogram Equalization (MBPHE)}

In this technique, initially it is divided into two main groups. They are bisections MBPHE and multi-sections MBPHE. Simplest group of MBPHE are the bisections MBPHE. Basically, this technique decomposes the input histogram into two sections. Therefore both the histogram sections are then equalized separately. Nevertheless, mean brightness can be preserved up to certain amount by bisections MBPHE. But at some cases, it needs a higher degree of preservation to eliminate unpleasant artifacts. Moreover, if the original histogram has a quasi-symmetrical distribution across its differentiating point then only bisections MBPHE can preserve the original mean brightness. But it cannot be found on most of the input histograms. So, in real life venture bisections MBPHE leads to the failure of preserving the mean intensity.

In Multi-sections MBPHE, a better mean brightness preservation operation is occurred as compared with the group of bisections MBPHE. In this technique the input histogram decomposes into $\mathrm{R}$ sub histograms, where $\mathrm{R}$ is any positive integer value. Thereafter independent equalization is performed on each sub-histogram. Furthermore, sub histograms formed continuously and repeatedly on the basis of mean or median intensity value or on the basis of input histogram shape.

These methods are very complex in detection of the differentiating point as well as it needs much computational time. Moreover, these methods also require high hardware requirements for consumer electronic implementations. Also, enhancement could not be achieved from these methods most of the time as it emphasizes more on restraining the mean intensity value [20].

\subsection{Brightness preserving weighted dynamic range histogram equalization (BPWDRHE)}

In this technique, histogram of the input image is separated by Otsu method. The reason behind this separation is to decreasing the errors dealt with sub histograms when its mean brightness separate HE process is undergone. Thus make it reliable, it requires changing the dynamic range based on its scale factor. Thereafter, smoothed histogram is obtained by HE. So, it can be normalized for keeping and maintaining the overall brightness with enhancing visualization effect [21]. The overall operation is performed in four steps:

Step1: Evaluating separating points and defines sub-histogram range.

Step2: Mapping the sub histograms based on adaptive scale factor.

Step3: Separate HE is processed for each resized subhistogram.
Step4: Smoothen histogram obtained from HE and maintain the overall brightness.

\section{CONCLUSIONS}

In this paper, various contrast enhancement techniques have been reviewed which are based on HE. Basically, HE is a simple and effective method but it cannot be implemented directly on consumer electronics as it causes variation in mean brightness and deterioration in the resultant image. So the major goal of all these $\mathrm{HE}$ techniques is to remove the drawbacks of $\mathrm{HE}$ and produce images without severe side effects, at the same time maintain maximum input mean brightness. In future, most of these techniques can be modified and used in consumer electronics for better clarity and enhanced images with less hardware implementation.

\section{RERERENCES}

[1] R. C. Gonzalez and R. E. Woods, "Digital image processing," $3^{\text {rd }}$ Edition, Prentice Hall, 2007.

[2] S.M. Pizer, E.P. Amburn, J.D. Austin, R. Cromartie, A. Geselowitz, T.Greer, B.H. Romeny, J.B. Zimmerman and K. Zuiderveld, "Adaptive histogram equalization and its variations", Computer Vision, Graphics and Image Processing, Vol. 39, pp.355-368, 1987.

[3] S.M. Pizer, R.E. Johnston, J.P. Ericksen, B.C. Yankaskas and K.E. Muller, "Contrast limited adaptive histogram equalization: speed and effectiveness", IEEE Conference on Visualization in Biomedical Computing (VBC '90), pp. 337-345, 1990 .

[4] Y.T. Kim, "Contrast enhancement using brightness preserving bi-histogram equalization", IEEE Transactions on Consumer Electronics, Vol. 43, pp.1-8, 1997.

[5] Y. Wang, Q. Chen and B. Zhang, "Image enhancement based on equal area dualistic sub-image histogram equalization method", IEEE Transactions on Consumer Electronics, Vol. 45, pp.68-75, 1999.

[6] S.D. Chen and A. Ramli, "Contrast enhancement using recursive mean separate histogram equalization for scalable brightness preservation," IEEE Transactions on Consumer Electronics, Vol. 49, pp.1301-1309, 2003.

[7] S.D. Chen and A. Ramli, "Minimum mean brightness error bi-histogram equalization in contrast enhancement", IEEE Transactions on Consumer Electronics, Vol. 49, pp.1310-1319, 2003.

[8] C. Wang and Z. Ye, "Brightness preserving histogram equalization with maximum entropy: a variational perspective", IEEE Transactions on Consumer Electronics, Vol. 51, pp.1326-1334, 2005.

[9] M.A. Wadud, H. Kabir, M.A. Dewan and O. Chae, "A dynamic histogram equalization for image contrast enhancement", IEEE Transactions on Consumer Electronics, Vol. 53, pp.593-599, 2007.

[10] Q. Wang and R.K. Ward, "Fast image/video contrast enhancement based on weighted threshold histogram equalization", IEEE Transactions on Consumer Electronics, Vol. 53, pp.757-764, 2007.

[11] K. Sim, C. Tso and Y. Tan, "Recursive sub-image histogram equalization applied to gray scale images", Pattern Recognition Letters, Vol. 28, pp.1209-1221, 2007. 
[12] H. Ibrahim and N.S. Kong, "Brightness preserving dynamic histogram equalization for image contrast enhancement", IEEE Transactions on Consumer Electronics, Vol. 53, pp.1752-1758, 2007.

[13] N. Sengee and H.K. Choi, "Brightness preserving weight clustering histogram equalization", IEEE Transactions on Consumer Electronics, Vol. 54, pp.1329-1337, 2008.

[14] M. Kim and M. G. Chung, "Recursively separated and weighted histogram equalization for brightness preservation and contrast enhancement", IEEE Transactions on Consumer Electronics, Vol. 54, pp.13891497, 2008.

[15] G.H. Park, H.H. Cho and M.R. Choi, "A contrast enhancement method using dynamic range separate histogram equalization", IEEE Transactions on Consumer Electronics, Vol. 54, pp.1981-1987, 2008.

[16] C.H. Lu, H.Y. Hsu and L. Wang, "A new contrast enhancement technique by adaptively increasing the value of histogram", IEEE International Workshop on Imaging Systems and Techniques, pp.407-411, 2009.
[17] T. Arici, S. Dikbas and Y. Altunbasak, "A histogram modification framework and its application for image contrast enhancement", IEEE Transactions on image processing, Vol. 18, pp.1921-1935, 2009.

[18] P. Rajavel, "Image dependent brightness preserving histogram equalization", IEEE Transactions on Consumer Electronics, Vol. 56, pp.756-763, 2010.

[19] C.H. Ooi and N.A. Isa, "Quadrants dynamic histogram equalization for contrast enhancement", IEEE Transactions on Consumer Electronics, Vol. 56, pp.25522559,2010

[20] M. Kaur, J. Kaur and J. Kaur, "Survey of contrast enhancement techniques based on histogram equalization", International Journal of Advanced Computer Science and Applications, Vol. 2, 2011.

[21] T. Huynh and T. Tien, "Brightness preserving weighted dynamic range histogram equalization for image contrast enhancement", IEEE International Conference on Advanced Technologies for Communications, pp.386391, 2013. 